\title{
Editorials
}

\section{The role of general practice in following up patients with trauma}

Worldwide, more than 5 million people die from injuries, accounting for $9 \%$ of the world's deaths. ${ }^{1}$ In the UK, falls and road traffic accidents are the most common causes of injury ${ }^{2}$ and GPs are often required to provide psychological support, manage, and coordinate follow up care. Patients with mild head trauma may only present to primary care $^{3}$ expecting to receive appropriate management and reassurance that their condition is self-limiting. This edition of the BJGP includes two papers highlighting the needs of patients and the challenges facing primary care physicians in following up injuries with both physical and psychological impacts.

The first linked paper describes the work of Theadom and colleagues from New Zealand (NZ) who investigated persistent problems in the year following diagnosis of mild traumatic brain injury (mTBI), resulting from head trauma. ${ }^{4}$ With regards to brain injury, the term 'mild' described patients post head injury who had a Glasgow Coma Scale (GCS) score of 13-15 or post traumatic amnesia within 24 hours of injury, ${ }^{3}$ however there is growing evidence that despite being labelled 'mild' the immediate effects of mTBI may have longer term consequences. In this study, 341 patients who had sustained an mTBI participated in comprehensive follow up assessments at regular intervals throughout the first year following injury. The assessments focused on post concussion symptoms, cognitive functioning, anxiety, depression, quality of life, and global functioning. Patients who did not attend hospital, and who may have been missed by previous studies, were specifically sought out. The results showed that nearly half (47.5\%) of participants experienced four or more mild to severe post-concussion symptoms at 1-year following the initial injury.

The most common symptoms at all follow up times were headaches, fatigue, forgetfulness, poor concentration, and taking longer to think (slower processing of information and recall). At 12 months, common symptoms also included sleep disturbance, irritability and feeling frustrated or impatient. Although these symptoms are often present in the general population, the frequency of memory difficulties, fatigue and irritability symptoms were far higher in the mTBI sample than in an NZ general population survey. A small percentage $(10 \%)$ of patients also had some impairment

\section{"The most common symptoms at all follow up times were headaches, fatigue, forgetfulness, poor concentration, and taking longer to think....}

in cognitive and global functioning higher than would be expected in the general population, but it is not possible to imply causality without details of pre injury ability and functioning, or assign a decrease in cognitive functioning to the injury itself rather than post-traumatic symptoms such as sleep disturbance. Interestingly, levels of depression, anxiety, and quality of life were found to be equivalent to general population mean scores.

The second linked paper is a qualitative study of patients' experiences following discharge from hospital after sustaining an unintentional injury. ${ }^{5}$ This was a nested study as part of multicentre longitudinal quantitative study assessing the impact of injuries on physical, psychological, social, and occupational functioning. ${ }^{2}$ Christie and colleagues interviewed 45 patients at various points in the year post injury, and 40 service providers (SPs). SPs included nurses, physiotherapists, doctors, and a number of other allied health professionals. Only three GPs were included in the sample. The results highlighted the complex needs of patients discharged post injury and areas where healthcare professionals could improve the experience for patients, such as providing sufficient information about the injury, its recovery, pain control, or help with returning to work. Other issues identified were a difficulty in accessing services such as physiotherapy in a time-sensitive manner and incongruence between what patients expect and what GPs deem necessary in terms of psychological support and access to counselling. Poor communication between secondary and primary care such as delayed or inadequate discharge letters, and a perceived lack of awareness that GPs have for available resources were also highlighted by SPs.

\section{SHORT AND LONG TERM NEEDS OF PATIENTS POST INJURY}

So what does this mean for general practice? Both papers identify the need for primary care professionals to be aware of the short term needs and longer term follow up for patients who have sustained an injury. In the short term, patients need psychological, physical and practical support. They need help understanding what has happened and their projected recovery, adequate symptom control, and a joined up health care system supporting timely access to rehabilitation. Although budget restraints or provision of services may affect local access to therapies, simply meeting regularly with patients soon after the injury, providing detailed verbal and written information and reassurance, ${ }^{6}$ and suggesting practical solutions and strategies on how to cope with symptoms or a gradual return to work can help reduce psychological distress and promote recovery. ${ }^{7}$ Ponsford et al demonstrated that giving patients who had sustained a minor head injury an information booklet 1 week post injury resulted in significantly lower rates of stress at 3 months than those who did not receive the booklet. ${ }^{8} \mathrm{GPs}$ should also be aware of predictors of poorer outcome following head injury such as being female or of non-Caucasian identity las in the NZ study), or factors associated with symptom prolongation such as a lack of social/family support, a concomitant orthopaedic or soft tissue injury, ongoing litigation or evolving depression, and anxiety at baseline.? 


\section{PHYSICAL EFFECTS OF INJURY}

The risk of pituitary dysfunction following TBI lpost traumatic hypopituitarism [PTHP]) is also well described and may affect as many has $30-80 \%$ of patients following TBI. ${ }^{9}$ Although current NICE guidelines in this area are lacking, there are recommendations that GPs should initiate baseline hormone evaluation at 3-6 months following any severity of $\mathrm{TBI}$, especially in patients with symptoms such as depression, cognitive impairment, and emotional lability, and involve an endocrinologist if there is any suggestion of hypopituitarism. ${ }^{9}$ GPs should also be alert to the possibility of epilepsy following $\mathrm{mTBI},{ }^{10}$ as well as dealing with chronic pain and potential disability.

\section{PSYCHOLOGICAL EFFECTS OF INJURY}

With regards to psychological issues, in the short (first 3-6 months) and longer term patients may need support to deal with both the trauma of the injury itself as well as persisting symptoms as a result of the injury. Injury, as well as other psychological risk factors such as peritraumatic emotional responses, that is, those individuals experiencing intensely negative emotional responses during or immediately after the index traumatic event, reported appreciably higher levels of post traumatic stress disorder (PTSD) symptoms. A metaanalysis of 2647 studies identified that these psychological processes, typically presenting as fear, helplessness, guilt, and shame, rather than prior characteristics, are the strongest predictors of PTSD. ${ }^{11}$ Similar studies have also identified a lack of social support as a major risk factor..$^{12}$ Therefore, asking patients about the 'meaning' of their experience and assessing their levels and quality of social support can yield useful predictors of further psychological distress. Early referral can lead to more positive psychological outcomes as once cognitive and behavioural avoidance become more marked, clinical presentations become more chronic and entrenched thus becoming more resistant to psychological approaches as recommended by NICE. This not only has implications for the need for earlier identification and referral, but for the accessibility, acceptance, adherence to treatment, and viability of recommended clinical interventions under the NICE guidelines which support the use of trauma focussed cognitive behavioural therapy (TFCBT) and antidepressants as an adjunct for PTSD. ${ }^{12}$

Post-traumatic stress disorder represents an excessive health and economic burden on patients, families, healthcare workers, hospitals, and society as a whole. Its effects extend far beyond the healthcare sector, and affect the quality of life as well as the ability to function socially and occupationally. The economic and social impact of PTSD is felt not only by those who experience the disorder, but also by families, co-workers, employers, and the wider society. ${ }^{13}$ The NICE cost impact report for CG26 on PTSD ${ }^{14}$ suggests a population prevalence of PTSD in England of around $1.5 \%$. However, commissioners need also be aware that the prevalence is likely to be higher for the reasons given above.

Finally, GPs can also find support from charities such as Headway and The United Kingdom Acquired Brain Injury Forum (UKABIF), which have online resources for professionals including guidelines for referrals to neuropsychological services for patients who have sustained an mTBI. The After Trauma website (www.aftertrauma. org), lists charities and useful organisations which offer invaluable emotional and practical support for patients and families whose lives have been affected by any type of injury. In summary, both papers in this issue highlight the short and long-term psychological and physical effects of injury. GPs have a vital role to play at all stages,

\section{REFERENCES}

1. World Health Organisation. Injuries and violence: the facts 2014. http://www.who.int/ violence_injury_prevention/media/news/2015/ Injury violence facts 2014/en/ laccessed 1 Dec 2015).

2. Kendrick D, O'Brien C, Christie N, et al. The impact of injuries study. multicentre study assessing physical, psychological, social and occupational functioning post injury - a protocol. BMC Public Health 2011; 11: 963.

3. Feigin VL, Theadom A, Barker-Collo S, et al. Incidence of traumatic brain injury in New Zealand: a population-based study. Lancet Neurol 2013; 12(1): 53-64

4. Theadom A, Parag V, Dowell T, et al. Persistent problems 1 year after mild traumatic brain injury. Br J Gen Pract 2016; DOI: 10.3399/ bjgp16X683161.

5. Christie, Beckett K, Earthy S, et al. Seeking support after hospitalisation for injury: a nested qualitative study of the role of primary care. $\mathrm{Br}$ J Gen Pract 2016; DOI: 10.3399/bjgp15X688141.

6. Kellezi B, Beckett K, Earthy S, et al. Understanding and meeting information needs following unintentional injury: comparing the accounts of patients, carers and service providers. Injury 2015; 46(4): 564-571.

7. Dhawan P, Rose A, Krassioukov A, Miller WC Early interventions for mild traumatic brain injury: Reflections on experience. BCMJ 2006; 48(9): 442-446

8. Ponsford J, Willmott C, Rothwell A, et al. Impact of early intervention on outcome

\section{ADDRESS FOR CORRESPONDENCE}

\section{Agnelo Fernandes}

Clinical Innovation and Research Centre (CIRC), Royal College of General Practitioners, 30 Euston Square, London NW1 2FB, UK.

E-mail: agnelo.fernandesanhs.net

including initiating referral to secondary care specialities as well as a maintaining regular contact with injured patients and their families. ${ }^{14}$

Agnelo Fernandes,

Urgent Care Lead, Royal College of General Practitioners, London.

\section{Lucy McDonnell,}

Visiting Research Associate and GP, King's College London.

\section{Stephen Regel,}

Professor, The Centre for Trauma, Resilience and Growth, Nottinghamshire Healthcare NHS Foundation Trust, University of Nottingham, Nottingham.

\section{Provenance}

Commissioned; not externally peer reviewed.

DOI: 10.3399/bjgp16X683065

following mild head injury in adults. J Neurol Neurosurg Psychiatry 2002; 73(3): 330-332.

9. Guerrero AF, Alfonso A. Traumatic brain injury-related hypopituitarism: a review and recommendations for screening combat veterans. Mil Med 2010; 175(8): 574-580.

10. Yeh CC, Chen TL, Hu CJ, et al. Risk of epilepsy after traumatic brain injury: a retrospective population-based cohort study. J Neurol Nuerosurg Psychiatry 2013; 84(4): 441-445.

11. Ozer EJ, Best SR, Lipsey TL, Weiss DS. Predictors of posttraumatic stress disorder and symptoms in adults: a meta-analysis. Psychol Bull 2003; 129(1): 52-73.

12. Brewin CR, Andrews B, Valentine JD. Metaanalysis of risk factors for posttraumatic stress disorder in trauma-exposed adults. J Consult Clin Psychol 2000; 68(5): 748-766.

13. McCrone P, Knapp M, Cawkill P. Posttraumatic stress disorder (PTSD) in the Armed Forces: health economic considerations. J Trauma Stress 2003; 16(5): 519-522.

14. National Institute for Health and Clinical Excellence. Post-traumatic stress disorder (PTSD). The management of PTSD in adults and children in primary and secondary care. National cost-impact report. Implementing NICE CG26. NICE 2005. https://www.nice.org uk/guidance/cg26/resources/cost-impactreport-193399453 laccessed 1 Dec 2015).

15. Donovan J, Cancelliere C, Cassidy JD. Summary of the findings of the International Collaboration on Mild Traumatic Brain Injury Prognosis. Chiropr Man Therap 2014; 22(1): 38. 